\title{
Consecutive Tandem Cycloaddition between Nitriles and Azides; Synthesis of 5-Amino-1H-[1,2,3]-triazoles
}

Ana T. P. C. Gomes, ${ }^{\mathrm{a}, \mathrm{b}}$ Priscila R. C. Martins, ${ }^{\mathrm{a}}$ David R. Rocha, ${ }^{\mathrm{a}}$ Maria G. P. M. S. Neves, ${ }^{\mathrm{b}}$ Vitor F. Ferreira, ${ }^{\mathrm{a}}$ Artur M.

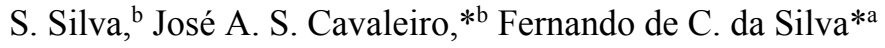

${ }^{a}$ Universidade Federal Fluminense, Instituto de Química, Departamento de Química Orgânica, 24020-150 Niterói, RJ, Brazil Fax +55(21)26292138; E-mail: gqofernando@vm.uff.br

b QOPNA, Department of Chemistry, University of Aveiro, 3810-193 Aveiro, Portugal Fax +351(234)370084; E-mail: jcavaleiro@ua.pt

Received: 19.09.2012; Accepted after revision: 08.11.2012

Abstract: Novel 5-amino-1H-1,2,3-triazoles were synthesized by a new synthetic route that involves consecutive tandem cycloaddition between nitriles and azides.

Key words: triazoles, tandem cycloaddition, consecutive reaction, nitriles, azides

Triazoles are biologically significant compounds not yet found in any natural source. ${ }^{1,2}$ However, the triazole moiety can be found in several bioactive compounds that act as potential new drugs to treat several diseases; some examples of significant 1,2,3-triazoles are shown in Figure 1. The acyl-hydrazone derivatives $\mathbf{1}$ and $\mathbf{2}$ have shown, respectively, excellent activity against Mycobacterium tuberculosis H37Rv (ATCC 27294) ${ }^{3}$ and strong inhibitory antiplatelet activity. ${ }^{4}$ The glycoconjugates $\mathbf{3}$ and $\mathbf{4}$ showed good inhibition respectively against $\alpha$-glucosidase with possible use in the treatment ${ }^{5}$ of diabetes mellitus type II and in vitro inhibitory profiles against HIV-1 reverse transcriptase. $^{6}$

In particular, it has been observed that 5-amino-1,2,3-triazoles and derivatives have important pharmacological properties such as antiallergic, ${ }^{7}$ antibacterial activity, ${ }^{8}$ and act as potassium channel activators ${ }^{9}$ or $\mathrm{A} 2 \mathrm{~A}$ adenosine receptor antagonists. ${ }^{10}$

The search for new biologically active triazole derivatives remains a great challenge. The 1,3-dipolar cycloaddition of azides with alkynes is a most versatile and popular route for the synthesis of 1,2,3-triazoles. ${ }^{11}$

The synthetic procedure initially developed by Huisgen ${ }^{12,13}$ involves long reaction times, high temperatures, and leads to the formation of mixtures of 1,4- and 1,5-regioisomers. However, the discovery of the $\mathrm{Cu}(\mathrm{I})$ catalyzed azide-alkyne cycloaddition reaction by Meldal $^{14}$ and Sharpless ${ }^{15}$ brought significant improvements to the synthesis of 1,2,3-triazoles. The combination of substituents on terminal alkynes and azide derivatives allows the synthesis of a range of 1,2,3-triazoles with es-

SYNLETT 2013, 24, 0041-0044

Advanced online publication: 06.12.2012

DOI: 10.1055/s-0032-1317712; Art ID: ST-2012-D0795-L

(C) Georg Thieme Verlag Stuttgart $\cdot$ New York

ter, hydroxyl, keto, aryl, haloalkyl, trimethylsilyl, phenylsulfonyl, and phosphonate groups. ${ }^{16-19}$ Despite being a very versatile reaction, it does not cover the entire range of 1,2,3-triazole compounds, such as certain amino derivatives.

Usually, the syntheses of 5-amino-1,2,3-triazoles are carried out from 1,5-regiospecific 1,3-dipolar cycloadditions between azides and nitriles containing an activated methylene group (e.g., electron-withdrawing groups such as cyano, ${ }^{20}$ phenyl, ${ }^{21}$ and carboxyl groups $\left.{ }^{22}\right)$ in a sodium alkoxide/alcohol medium.
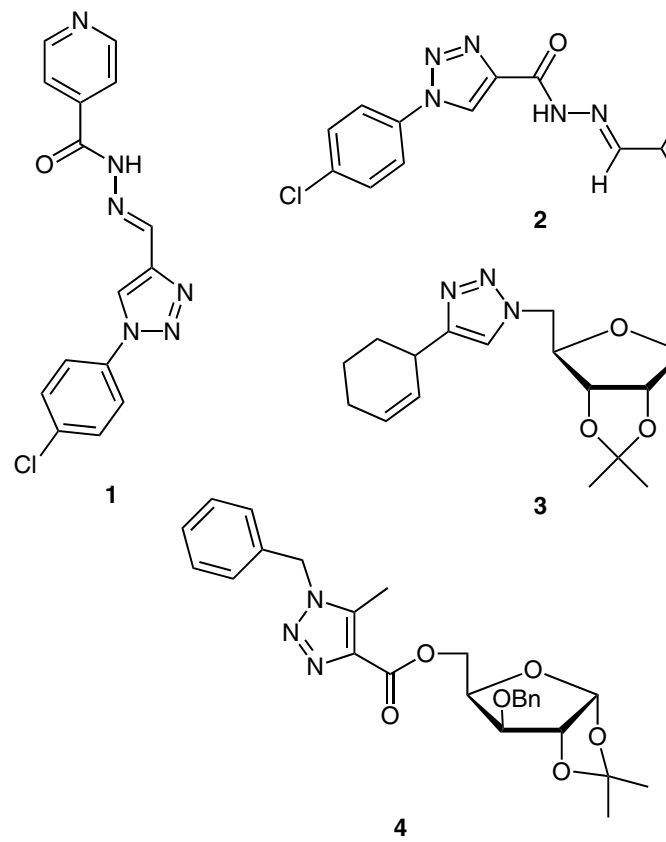

Figure 1 Structure of some $1 H$-1,2,3-triazoles that have displayed significant biological applications

Other methods are available for the synthesis of 1,2,3-triazoles such as the condensation of alkyl diazoacetoacetates with phenyl-substituted hydrazines, ${ }^{23,24}$ condensation of $\alpha$-diazocompounds with amines followed by electrocyclization, ${ }^{25,26}$ cyclization of cyano-2-aryl-hydrazones, ${ }^{27}$ coupling of allyl carbonates with trimethylsilyl azide catalyzed by $\left[\mathrm{Pd}_{2}(\mathrm{dba})_{3}\right],{ }^{28} \mathrm{NH}$-arylation 
catalyzed by palladium, ${ }^{29}$ and addition and cyclization between ethyl cyanoacetate and aromatic azides. ${ }^{30}$

Based on our interest in the establishment of new synthetic methodologies leading to new potential bioactive $1 H$ 1,2,3-triazoles, the work presented herein describes the synthesis of 5-amino- $1 H$-1,2,3-triazoles by consecutive tandem cycloaddition between nonactivated nitriles and azides. Scheme 1 shows a comparison between literature data and the present studies on the synthesis of 5-amino1,2,3-triazoles.

(a)

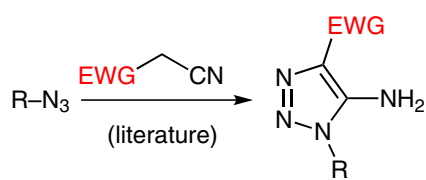

(b)

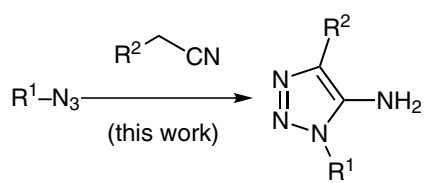

$\mathrm{R}^{1}=\mathrm{Ph}, 4-\mathrm{ClC}_{6} \mathrm{H}_{4}, 4-\mathrm{MeOC}_{6} \mathrm{H}_{4}$,

$$
\mathrm{R}^{2}=\mathrm{H}, \mathrm{Me}
$$

Scheme 1 Comparison between literature data (a) and the present studies (b) on the synthesis of 5-amino-1,2,3-triazoles

Azide derivatives 5 were prepared according to the literature procedure. ${ }^{3}$ The reactions of the lithium carbanion, generated by the addition of BuLi to alkyl nitriles $\mathbf{6}$, with azides 5 gave rise to the new 5-amino- $1 H$-1,2,3-triazoles $(7 \mathbf{a}-\mathbf{h})$ in good yields (Scheme 2). ${ }^{31}$ The structures of these compounds were assigned on the basis of their ${ }^{1} \mathrm{H}$ and ${ }^{13} \mathrm{C}$ NMR spectra, and their molecular compositions were confirmed by HRMS (ESI) analysis. ${ }^{32}$
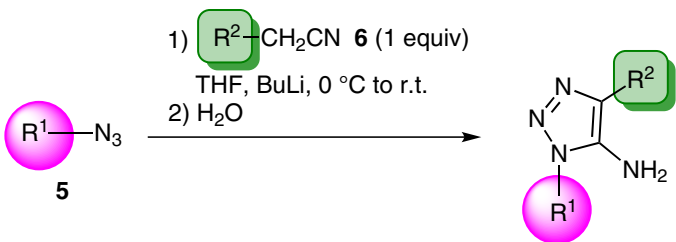

$$
\begin{aligned}
& \text { 7a, } R^{1}=\mathrm{Ph}, \mathrm{R}^{2}=\mathrm{H}, 84 \% \\
& \text { 7b, } \mathrm{R}^{1}=\mathrm{Ph}, \mathrm{R}^{2}=\mathrm{Me}, 61 \% \\
& \text { 7c, } \mathrm{R}^{1}=4-\mathrm{ClC}_{6} \mathrm{H}_{4}, \mathrm{R}^{2}=\mathrm{H}, 82 \% \\
& \text { 7d, } \mathrm{R}^{1}=4-\mathrm{ClC}_{6} \mathrm{H}_{4}, \mathrm{R}^{2}=\mathrm{Me}, 76 \% \\
& \text { 7e, } \mathrm{R}^{1}=4-\mathrm{MeOC}_{6} \mathrm{H}_{4}, \mathrm{R}^{2}=\mathrm{H}, 85 \% \\
& \text { 7f, } \mathrm{R}^{1}=4-\mathrm{MeOC}_{6} \mathrm{H}_{4}, \mathrm{R}^{2}=\mathrm{Me}, 86 \% \\
& \text { 7g, } \mathrm{R}^{1}=\text { galactopyranosyl, } \mathrm{R}^{2}=\mathrm{H}, 82 \% \\
& \text { 7h, } \mathrm{R}^{1}=\text { galactopyranosyl, } \mathrm{R}^{2}=\mathrm{Me}, 54 \%
\end{aligned}
$$

Scheme 2 Synthesis of 5-amino-1H-1,2,3-triazoles 7a-h

However, when the reaction was performed under the same conditions but with an excess of acetonitrile, ${ }^{33}$ new triazole derivatives 8a-c were formed (Scheme 3). All the obtained compounds were fully characterized by ${ }^{1} \mathrm{H}$ and ${ }^{13} \mathrm{C}$ NMR spectroscopy and HRMS (ESI) analysis. ${ }^{34}$

The formation of compounds $\mathbf{8 a}-\mathbf{c}$ results from the addition of a second acetonitrile molecule to C-4. This phenomenon was not observed when the reaction was performed with propanonitrile since the $\mathrm{C}-4$ position is blocked by the methyl group.

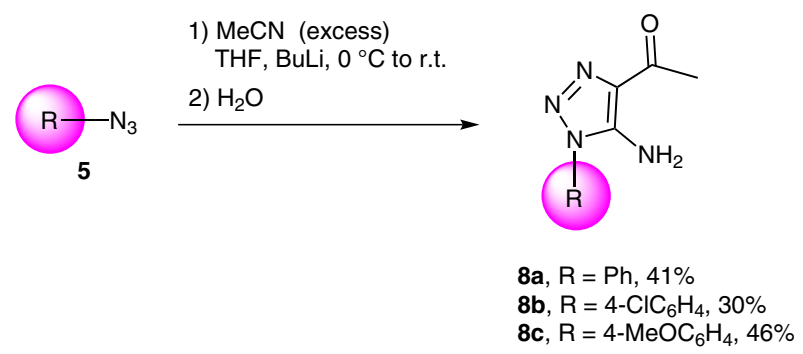

Scheme 3 Synthesis of 1-(5-amino-1H-1,2,3-triazol-4-yl)ethanone $\mathbf{8 a}-\mathbf{c}$

The proposed mechanism of this reaction is shown in Scheme 4. This mechanism involves the consecutive tandem cycloaddition of the lithium carbanion of the nitrile derivatives to the azides $\mathbf{5}$. When the reactions were performed with one equivalent of nitrile, the addition of water causes aromatization and formation of the amino group at position $\mathrm{C}-5$. When the reaction is conducted with an excess of acetonitrile, addition of a second acetonitrile molecule occurs after the cycloaddition, followed by the addition of water, leading to aromatization, the formation of the carbonyl group at C-4, and the amino group at C-5.

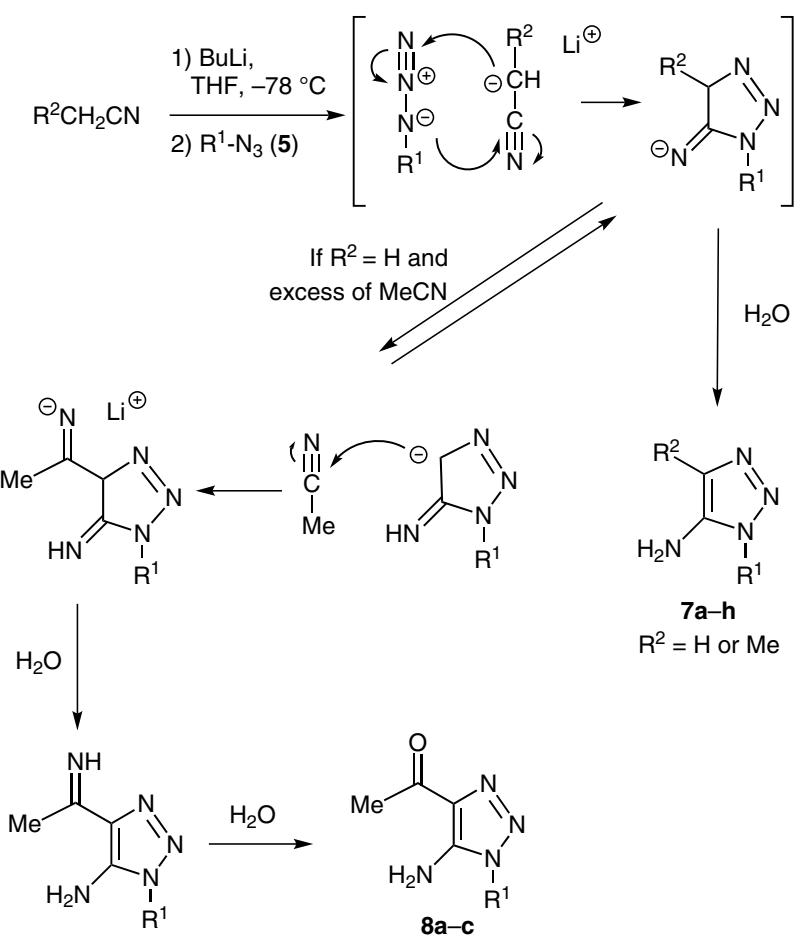

Scheme 4 Proposed mechanism for the formation of aminotriazoles $7 \mathbf{a}-\mathbf{h}$ and $\mathbf{8 a}-\mathbf{c}$ 
In conclusion, this study reports a new methodology with which to prepare novel 5-amino- $1 H$-1,2,3-triazoles involving consecutive tandem cycloaddition between nonactivated nitriles and azides. This approach allows the synthesis of 5-amino-1,2,3-triazoles that are not easily obtained by other procedures under mild conditions.

\section{Acknowledgment}

Thanks are due to the University of Aveiro (Aveiro, Portugal) and Universidade Federal Fluminense (Niterói, RJ, Brazil), CNPq, CAPES, FAPERJ and FCT/CAPES collaborative programme for funding. This work was partially supported by CNPq-FAPERJ. A.T.P.C.G. also thanks FCT for her research grant (SFRH/BPD/79521/2011).

\section{References and Notes}

(1) Melo, J. O. F.; Donnici, C. L.; Augusti, R.; Ferreira, V. F.; Souza, M. C. B. V.; Ferreira, M. L. G.; Cunha, A. C. Quim. Nova 2006, 29, 569.

(2) Sharma, P.; Kumar, A.; Upadhyay, S.; Singh, J.; Sahu, V. Med. Chem. Res. 2010, 19, 589.

(3) Boechat, N.; Ferreira, V. F.; Ferreira, S. B.; Ferreira, M. L. G.; da Silva, F. C.; Bastos, M. M.; Costa, M. S.; Lourenço, M. C. S.; Pinto, A. C.; Krettli, A. U.; Aguiar, A. C.; Teixeira, B. M.; da Silva, N. V.; Martins, P. R. C.; Bezerra, F. A. F. M.; Camilo, A. L. S.; da Silva, G. P.; Costa, C. C. P. J. Med. Chem. 2011, 54, 5988.

(4) Cunha, A. C.; Figueiredo, J. M.; Tributino, J. L. M.; Miranda, A. L. P.; Castro, H. C.; Zingali, R. B.; Fraga, C. A. M.; de Souza, M. C. B. V.; Ferreira, V. F.; Barreiro, E. J. Bioorg. Med. Chem. 2003, 11, 2051.

(5) Ferreira, S. B.; Sodero, A. C. R.; Cardoso, M. F. C.; Lima, E. S.; Kaiser, C. R.; Silva, F. P. Jr.; Ferreira, V. F. J. Med. Chem. 2010, 53, 2364.

(6) da Silva, F. C.; de Souza, M. C. B. V.; Frugulhetti, I. C. P. P.; Castro, H. C.; Souza, S. L. O.; Souza, T. M. L.; Rodrigues, D. Q.; de Souza, A. M. T.; Abreu, P. A.; Passamani, F.; Rodrigues, C. R.; Ferreira, V. F. Eur. J. Med. Chem. 2009, 44, 373 .

(7) (a) da Settimo, A.; Livi, O.; Ferrarini, P. L.; Biagi, G. Farmaco, Ed. Sci. 1980, 35, 308. (b) da Settimo, A.; Livi, O.; Ferrarini, P. L.; Primofiore, G. Farmaco, Ed. Sci. 1980, 35, 298.

(8) Zhang, Z. Y.; Liu, Y.; Zheng, G. Y.; Chen, M. Q.; Yang, S. Y. Acta Pharm. Sin. 1991, 26, 809.

(9) Biagi, G.; Calderone, V.; Giorgi, I.; Livi, O.; Scartoni, V.; Baragatti, B.; Martinotti, E. Eur. J. Med. Chem. 2000, 35, 715.

(10) Baraldi, P. G.; Cacciari, B.; Spalluto, G.; De Las Infants, M. J. P.; Zocchi, C.; Ferrara, S.; Dionisotti, S. Farmaco, Ed. Sci. 1996, 51, 29.

(11) Freitas, L. B. O.; Ruela, F. A.; Pereira, G. R.; Alves, R. B.; Freitas, R. P.; Santos, L. J. Quim. Nova 2011, 34, 1791.

(12) Huisgen, R. Angew. Chem., Int. Ed. Engl. 1963, 2, 565.

(13) Huisgen, R.; Szeimies, G.; Moebius, L. Chem. Ber. 1967, 100, 2494

(14) Tornøe, C. W.; Christensen, C.; Meldal, M. J. Org. Chem. 2002, 67, 3057.

(15) Rostovtsev, V. V.; Green, L. G.; Fokin, V. V.; Sharpless, K. B. Angew. Chem. Int. Ed. 2002, 41, 2596.

(16) Biagi, G.; Giorgi, I.; Livi, O.; Lucacchini, A.; Martini, C.; Scartoni, V. J. Pharm. Sci. 1993, 82, 893.

(17) Palacios, F.; Ochoa de Retana, A. M.; Pagalday, J. Heterocycles 1994, 38, 95.
(18) Boyer, J. H.; Mack, C. H.; Goebel, N.; Morgan, L. R. Jr. J. Org. Chem. 1958, 23, 1051.

(19) Tanaka, Y.; Miller, S. I. J. Org. Chem. 1973, 38, 2708.

(20) (a) Smith, C. J.; Nikbin, N.; Ley, S. V.; Lange, H.; Baxendale, I. R. Org. Biomol. Chem. 2011, 9, 1938. (b) Rozhkov, V. Y.; Batog, L. V.; Shevtsova, E. K.; Struchkova, M. I. Mendeleev Commun. 2004, 14, 76.

(21) (a) Lieber, E.; Chao, T. S.; Rao, C. N. R. Org. Synth. 1957, 37, 26. (b) Smith, P. A. S.; Friar, J. J.; Resemann, W.; Watson, A. C. J. Org. Chem. 1990, 55, 3351. (c) L'Abbe, G.; Hassner, A. J. Heterocycl. Chem. 1970, 7, 361.

(22) L'Abbe, G.; Beenaerts, L. Tetrahedron 1989, 45, 749.

(23) Jordão, A. K.; Ferreira, V. F.; Souza, T. M. L.; Faria, G. G. S.; Machado, V.; Abrantes, J. L.; da Souza, M. C. B. V.; Cunha, A. C. Bioorg. Med. Chem. 2011, 19, 1860.

(24) Campos, V. R.; Abreu, P. A.; Castro, H. C.; Rodrigues, C. R.; Jordão, A. K.; Ferreira, V. F.; de Souza, M. C. B. V.; Santos, F. C.; Moura, L. A.; Domingos, T. S.; Carvalho, C.; Sanchez, E. F.; Fuly, A. L.; Cunha, A. C. Bioorg. Med. Chem. 2009, 17, 7429.

(25) Dabak, K.; Sezer, Z.; Akar, A.; Anac, O. Eur. J. Med. Chem. 2003, 38, 215.

(26) Costa, M. S.; Boechat, N.; Rangel, E. A.; da Silva, F. C.; de Souza, A. M. T.; Rodrihuez, C. R.; Castro, H. C.; Junior, I. N.; Lourenço, M. C. S.; Wardell, S. M. S. V.; Ferreira, V. F. Bioorg. Med. Chem. 2006, 14, 8644.

(27) Ghoslan, S. A. S.; Abdelhamid, I. A. A.; Ibrahin, H. M.; Elnagdi, M. H. ARKIVOC 2006, (xv), 53.

(28) Kamijo, S.; Jin, T.; Huo, Z.; Yamamoto, Y. Tetrahedron Lett. 2002, 43, 9707.

(29) Ueda, S.; Su, M.; Buchwald, S. L. Angew. Chem. Int. Ed. 2011, 50, 8944.

(30) Liu, H.; He, X.; Choi, H. S.; Yang, K.; Woodmansee, D.; Wang, Z.; Ellis, D. A.; Wu, B.; He, Y. PCT Int. Appl. WO 2006047516-A2, 2006, 259.

(31) Preparation of Compounds $\mathbf{7 a}-\mathbf{h}$; General Procedure: To a solution of $\mathrm{BuLi}(2 \mathrm{mmol})$ in anhydrous THF $(1.5 \mathrm{~mL})$, was added a solution of the nitrile (acetonitrile or propionitrile; $2 \mathrm{mmol})$ in anhydrous THF $(2 \mathrm{~mL})$ at $0{ }^{\circ} \mathrm{C}$ and under an argon atmosphere. After $5 \mathrm{~min}$, a solution of azide derivative $\mathbf{5}(2 \mathrm{mmol})$ in anhydrous THF $(2 \mathrm{~mL})$ was added. The reaction was allowed to warm to r.t. and monitored by TLC. Distilled $\mathrm{H}_{2} \mathrm{O}(10 \mathrm{~mL})$ was then added and the mixture was extracted with EtOAc. The organic layer was dried with sodium sulfate, filtered, and evaporated under reduced pressure. The residue was purified by column chromatography on silica gel eluting with increasing polarity gradient mixture of hexane and EtOAc.

(32) 1-Phenyl-1H-1,2,3-triazole-5-amine (7a): Yield: $84 \%$; white solid; $\mathrm{mp} 107.4-108.2^{\circ} \mathrm{C}$. IR (KBr): $3446(\mathrm{~N}-\mathrm{H}) \mathrm{cm}^{-1}$; ${ }^{1} \mathrm{H} \mathrm{NMR}\left(\mathrm{CDCl}_{3}-\mathrm{MeOD}, 300 \mathrm{MHz}\right): \delta=5.64(\mathrm{br} \mathrm{s}, 2 \mathrm{H}$, $\mathrm{NH}_{2}$ ), 6.75 (s, $\left.1 \mathrm{H}, \mathrm{H}-4\right), 6.78-6.93$ (m, $\left.1 \mathrm{H}, \mathrm{H}-4^{\prime}\right), 7.07-$ $7.11\left(\mathrm{~m}, 2 \mathrm{H}, \mathrm{H}-3^{\prime}\right.$ and $\left.\mathrm{H}-5^{\prime}\right), 7.24-7.30$ (m, $\left.2 \mathrm{H}, \mathrm{H} 2^{\prime}-\mathrm{H}-6^{\prime}\right)$; ${ }^{13} \mathrm{C}$ NMR $\left(\mathrm{CDCl}_{3}-\mathrm{MeOD}, 75 \mathrm{MHz}\right): \delta=128.8(\mathrm{C}-\mathrm{Ph})$, 120.0 (C-2' and C-6'), 125.8 (C-4'), 130.1 (C-3' and C-5), 134.60, 135.5; HRMS (ESI): $m / z[\mathrm{M}+\mathrm{H}]^{+}$calcd for $\mathrm{C}_{8} \mathrm{H}_{9} \mathrm{~N}_{4}$ : 161.1833; found: 161.0924

4-Methyl-1-phenyl-1H-1,2,3-triazole-5-amine (7b): Yield: $61 \%$; white solid; mp $127.5-128.9^{\circ} \mathrm{C}$. IR (KBr): $3381(\mathrm{~N}-\mathrm{H}) \mathrm{cm}^{-1} ;{ }^{1} \mathrm{H} \mathrm{NMR}\left(\mathrm{CDCl}_{3}-\mathrm{MeOD}, 300 \mathrm{MHz}\right): \delta=$ $\delta 2.23\left(\mathrm{~s}, 3 \mathrm{H}, \mathrm{CH}_{3}\right), 3.82$ (br s, $\left.2 \mathrm{H}, \mathrm{NH}_{2}\right), 7.41-7.58(\mathrm{~m}$, $5 \mathrm{H}, \mathrm{H}-\mathrm{Ph}) ;{ }^{13} \mathrm{C} \mathrm{NMR}\left(\mathrm{CDCl}_{3}-\mathrm{MeOD}, 75 \mathrm{MHz}\right): \delta=9.5$ $\left(\mathrm{CH}_{3}\right), 123.8(\mathrm{C}-\mathrm{Ph}), 126.0,128.8(\mathrm{C}-\mathrm{Ph}), 129.6(\mathrm{C}-\mathrm{Ph})$, 135.60, 137.5; HRMS (ESI): $m / z[\mathrm{M}+\mathrm{H}]^{+}$calcd for $\mathrm{C}_{9} \mathrm{H}_{11} \mathrm{~N}_{4}$ : 175.2099; found: 175.0977.

1-(4-Chlorophenyl)-1 H-1,2,3-triazole-5-amine (7c): Yield: $82 \%$; brown solid; mp $118.6-119.7^{\circ} \mathrm{C}$. IR (KBr): 
$3297(\mathrm{~N}-\mathrm{H}), 831(\mathrm{C}-\mathrm{Cl}) \mathrm{cm}^{-1} ;{ }^{1} \mathrm{H}$ NMR (DMSO- $d_{6}, 500$ $\mathrm{MHz}): \delta=5.78$ (br s, $2 \mathrm{H}, \mathrm{NH}_{2}$ ), 7.06 (s, $\left.1 \mathrm{H}, \mathrm{H}-4\right), 7.75$ (s, $4 \mathrm{H}, \mathrm{H}-2^{\prime}, \mathrm{H}-3^{\prime}, \mathrm{H}-5^{\prime}$ and $\left.\mathrm{H}-6^{\prime}\right) ;{ }^{13} \mathrm{C} \mathrm{NMR}\left(\mathrm{CDCl}_{3}, 125\right.$ MHz): $\delta=116.97(\mathrm{C}-5), 125.31(\mathrm{C}-\mathrm{Ph}), 128.55\left(\mathrm{C}-1^{\prime}\right)$, 129.43 (C-Ph), 132.70 (C-6'), 134.58 (C-4'); HRMS (ESI): $m / z[\mathrm{M}+\mathrm{H}]^{+}$calcd for $\mathrm{C}_{8} \mathrm{H}_{8} \mathrm{ClN}_{4}$ : 195.6284; found: 195.0431

1-(4-Chlorophenyl)-4-methyl-1H-1,2,3-triazole-5-amine (7d): Yield: 76\%; yellow solid; mp $139.6-140.3{ }^{\circ} \mathrm{C}$; IR (KBr): $3340(\mathrm{~N}-\mathrm{H}), 834(\mathrm{C}-\mathrm{Cl}) \mathrm{cm}^{-1} ;{ }^{1} \mathrm{H}$ NMR $\left(\mathrm{CDCl}_{3}-\right.$ MeOD, $300 \mathrm{MHz}): \delta=2.25$ (s, $\left.3 \mathrm{H}, \mathrm{CH}_{3}\right), 7.51$ (s, $2 \mathrm{H}, \mathrm{H}-3^{\prime}$ and $\left.\mathrm{H}-5^{\prime}\right), 7.52$ (s, $2 \mathrm{H}, \mathrm{H}-2^{\prime}$ and $\left.\mathrm{H}-6^{\prime}\right) ;{ }^{13} \mathrm{C} \mathrm{NMR}\left(\mathrm{CDCl}_{3}-\right.$ $\mathrm{MeOD}, 75 \mathrm{MHz}): \delta=9.4\left(\mathrm{CH}_{3}\right), 124.9\left(\mathrm{C}-3^{\prime}\right.$ and $\left.\mathrm{C}-5^{\prime}\right), 126.4$ (C-5), 129.7 (C-2' and C-6'), 134.0 (C-4), $134.6\left(\mathrm{C}-1^{\prime}\right), 137.4$ (C-4'); HRMS (ESI): $m / z[\mathrm{M}+\mathrm{H}]^{+}$calcd for $\mathrm{C}_{9} \mathrm{H}_{10} \mathrm{ClN}_{4}$ : 209.6550; found: 209.0586 .

1-(1,2:3,4-Di-O-isopropylideno- $\alpha$-D-galactopyranosyl)$1 \mathrm{H}-1,2,3$-triazole-5-amine (7g): Yield: 82\%; yellow solid; mp 179.0-181.4 ${ }^{\circ} \mathrm{C}$; IR (KBr): $3410(\mathrm{~N}-\mathrm{H}), 2922$ (C-C sugar unit), 2853 (C-C sugar unit), 1002-1212 (C-O-C sugar unit) $\mathrm{cm}^{-1} ;{ }^{1} \mathrm{H} \mathrm{NMR}\left(\mathrm{CDCl}_{3}-\mathrm{MeOD}, 500 \mathrm{MHz}\right): \delta=1.28(\mathrm{~s}, 3 \mathrm{H}$, $\left.\mathrm{CH}_{3}\right), 1.36\left(\mathrm{~s}, 3 \mathrm{H}, \mathrm{CH}_{3}\right), 1.38\left(\mathrm{~s}, 3 \mathrm{H}, \mathrm{CH}_{3}\right), 1.51(\mathrm{~s}, 3 \mathrm{H}$, $\left.\mathrm{CH}_{3}\right), 4.14-4.25$ ( $\mathrm{m}, 2 \mathrm{H}, \mathrm{H}-5^{\prime}$ and $\left.\mathrm{H}-6^{\prime}\right), 4.26-4.40$ ( $\mathrm{m}, 2 \mathrm{H}$, H-4' and H-6'), 4.49 (dd, $\left.J=2.5,4.0 \mathrm{~Hz}, 1 \mathrm{H}, \mathrm{H}-2^{\prime}\right), 4.64$ $\left(\mathrm{dd}, J=7.8,2.5 \mathrm{~Hz}, 1 \mathrm{H}, \mathrm{H}-3^{\prime}\right), 5.50(\mathrm{~d}, J=4.0 \mathrm{~Hz}, 1 \mathrm{H}, \mathrm{H}-$ $\left.1^{\prime}\right), 7.04$ (s, $\left.1 \mathrm{H}, \mathrm{H}-4\right) ;{ }^{13} \mathrm{C} \mathrm{NMR}\left(\mathrm{CDCl}_{3}-\mathrm{MeOD}, 125 \mathrm{MHz}\right)$ : $\delta=24.4\left(\mathrm{CH}_{3}\right), 24.8\left(\mathrm{CH}_{3}\right), 25.9\left(\mathrm{CH}_{3}\right), 26.0\left(\mathrm{CH}_{3}\right), 49.6(\mathrm{C}-$ $\left.6^{\prime}\right), 68.6\left(\mathrm{C}-5^{\prime}\right), 70.4\left(\mathrm{C}-2^{\prime}\right), 70.6\left(\mathrm{C}-3^{\prime}\right), 71.1\left(\mathrm{C}-4^{\prime}\right), 96.1(\mathrm{C}-$ $\left.1^{\prime}\right), 109.3,109.8$; HRMS (ESI): $m / z[\mathrm{M}+\mathrm{H}]^{+}$calcd for $\mathrm{C}_{14} \mathrm{H}_{23} \mathrm{~N}_{4} \mathrm{O}_{5}$ : 327.3557; found: 327.1659 .

1-(1,2:3,4-Di- $O$-isopropylideno- $\alpha$-D-galactopyranosyl)-4methyl-1H-1,2,3-triazole-5-amine (7h): Yield: 54\%; white solid; mp 204.2-205.6 ${ }^{\circ} \mathrm{C}$; IR (KBr): 3450 (N-H), 2987 (C$\mathrm{C}$ sugar unit), 2851 (C-C sugar unit), 1070-1237 (C-O-C sugar unit) $\mathrm{cm}^{-1} ;{ }^{1} \mathrm{H} \mathrm{NMR}\left(\mathrm{CDCl}_{3}-\mathrm{MeOD}, 300 \mathrm{MHz}\right): \delta{ }^{1} \mathrm{H}$ $\operatorname{NMR}\left(\mathrm{CDCl}_{3}-\mathrm{MeOD}, 300 \mathrm{MHz}\right): \delta=1.36\left(\mathrm{~s}, 3 \mathrm{H}, \mathrm{CH}_{3}\right)$, $1.49\left(\mathrm{~s}, 3 \mathrm{H}, \mathrm{CH}_{3}\right), 1.55\left(\mathrm{~s}, 3 \mathrm{H}, \mathrm{CH}_{3}\right), 1.57\left(\mathrm{~s}, 3 \mathrm{H}, \mathrm{CH}_{3}\right)$, $2.34\left(\mathrm{~s}, 3 \mathrm{H}, \mathrm{CH}_{3}\right), 4.15-4.20\left(\mathrm{~m}, 1 \mathrm{H}, \mathrm{H}-5^{\prime}\right), 4.45-4.55(\mathrm{~m}$, $\left.2 \mathrm{H}, \mathrm{H}-6^{\prime}\right), 4.31$ (dd, $\left.J=7.9,1.9 \mathrm{~Hz}, 1 \mathrm{H}, \mathrm{H}-4^{\prime}\right), 4.34$ (dd, $J$ $\left.=5.0,2.5 \mathrm{~Hz}, 1 \mathrm{H}, \mathrm{H}-2^{\prime}\right), 4.65(\mathrm{dd}, J=7.9,2.6 \mathrm{~Hz}, 1 \mathrm{H}, \mathrm{H}-$ $\left.3^{\prime}\right), 5.58\left(\mathrm{~d}, J=5.0 \mathrm{~Hz}, 1 \mathrm{H}, \mathrm{H}-1^{\prime \prime}\right) ;{ }^{13} \mathrm{C} \mathrm{NMR}\left(\mathrm{CDCl}_{3}-\right.$ MeOD, $75 \mathrm{MHz}): \delta=24.3\left(\mathrm{CH}_{3}\right), 24.6\left(\mathrm{CH}_{3}\right), 25.6\left(\mathrm{CH}_{3}\right)$, $25.8\left(\mathrm{CH}_{3}\right), 44.4\left(\mathrm{C}-6^{\prime}\right), 65.6\left(\mathrm{C}-5^{\prime}\right), 70.4\left(\mathrm{C}-2^{\prime}\right), 70.6\left(\mathrm{C}-3^{\prime}\right)$, 70.8 (C-4'), 97.3 (C-1'), 107.9, 108.7; HRMS (ESI): $m / z$ [M $+\mathrm{H}]^{+}$calcd for $\mathrm{C}_{15} \mathrm{H}_{25} \mathrm{~N}_{4} \mathrm{O}_{5}: 341.3823$; found: 341.1695 .
(33) Preparation of 8a-c; General Procedure: To a solution of BuLi ( $2 \mathrm{mmol})$ in anhydrous THF $(1.5 \mathrm{~mL})$, was added a solution of the acetonitrile $(4 \mathrm{mmol})$ in anhydrous THF (2.0 $\mathrm{mL}$ ) at $0{ }^{\circ} \mathrm{C}$ under an argon atmosphere. After $5 \mathrm{~min}$, a solution of azide derivative $5(2 \mathrm{mmol})$ in anhydrous THF $(2.0 \mathrm{~mL})$ was added. The reaction was allowed to warm to r.t. with monitoring by TLC. Distilled water $(10 \mathrm{~mL})$ was then added, the mixture was extracted with EtOAc and the organic layer was dried with sodium sulfate, filtered, and evaporated under reduced pressure. The residue was purified by column chromatography on silica gel and eluted with increasing polarity gradient mixture of hexane and ethyl acetate.

(34) 4-Methyl-1-(5-amino-1-phenyl-1H-1,2,3-triazole-4yl)ethanone (8a): Yield: 41\%; white solid; $\mathrm{mp} 208.4$ $209.8^{\circ} \mathrm{C}$; IR (KBr): $3293(\mathrm{~N}-\mathrm{H}), 1664(\mathrm{C}=\mathrm{O}) \mathrm{cm}^{-1} ;{ }^{1} \mathrm{H}$ NMR $\left(\mathrm{CDCl}_{3}, 300 \mathrm{MHz}\right): \delta=2.16\left(\mathrm{~s}, 3 \mathrm{H}, \mathrm{CH}_{3}\right), 7.10(\mathrm{t}, J=$ $\left.7.9 \mathrm{~Hz}, 1 \mathrm{H}, \mathrm{H}-4^{\prime}\right), 7.30$ (d, $J=7.9 \mathrm{~Hz}, 2 \mathrm{H}, \mathrm{H}-3^{\prime}$ and $\left.\mathrm{H}-5^{\prime}\right)$, $7.50\left(\mathrm{~d}, J=7.9 \mathrm{~Hz}, 2 \mathrm{H}, \mathrm{H}-2^{\prime}\right.$ and $\left.\mathrm{H}-6^{\prime}\right), 7.60(\mathrm{br} \mathrm{s}, 2 \mathrm{H}$, $\left.\mathrm{NH}_{2}\right) ;{ }^{13} \mathrm{C} \mathrm{NMR}\left(\mathrm{CDCl}_{3}, 75 \mathrm{MHz}\right): \delta=24.5\left(\mathrm{CH}_{3}\right), 119.9(\mathrm{C}-$ $2^{\prime}$ and $\left.\mathrm{C}-6^{\prime}\right), 124.3\left(\mathrm{C}-4^{\prime}\right), 128.9\left(\mathrm{C}-3^{\prime}\right.$ and $\left.\mathrm{C}-5^{\prime}\right), 137.9$, 168.5; HRMS (ESI): $m / z[\mathrm{M}+\mathrm{H}]^{+}$calcd for $\mathrm{C}_{10} \mathrm{H}_{11} \mathrm{~N}_{4} \mathrm{O}$ : 203.2200; found: 203.1021 .

1-[5-Amino-1-(4-chlorophenyl)-1 H-1,2,3-triazole-4yl]ethanone (8b): Yield: $30 \%$; white solid; $\mathrm{mp} 210.7-$ $211.2{ }^{\circ} \mathrm{C}$; IR (KBr): $3359(\mathrm{~N}-\mathrm{H}), 1658(\mathrm{C}=\mathrm{O}) \mathrm{cm}^{-1} ;{ }^{1} \mathrm{H}$ $\operatorname{NMR}\left(\mathrm{CDCl}_{3}, 500 \mathrm{MHz}\right): \delta=2.64\left(\mathrm{~m}, 3 \mathrm{H}, \mathrm{COCH}_{3}\right), 5.66$ (s, $2 \mathrm{H}, \mathrm{NH}_{2}$ ), 7.49 (d, $J=7.5 \mathrm{~Hz}, 2 \mathrm{H}, \mathrm{H}-3^{\prime}$ and $\left.\mathrm{H}-5^{\prime}\right), 7.56$ $\left(\mathrm{d}, J=7.5 \mathrm{~Hz}, 2 \mathrm{H}, \mathrm{H}-2^{\prime}\right.$ and $\left.\mathrm{H}-6^{\prime}\right) ;{ }^{13} \mathrm{C}$ NMR $\left(\mathrm{CDCl}_{3}, 125\right.$ $\mathrm{MHz}): \delta=26.2\left(\mathrm{CH}_{3}\right), 125.0\left(\mathrm{C}^{\prime}\right.$ and $\left.\mathrm{C}-5^{\prime}\right), 129.6(\mathrm{C}-5)$, $130.3\left(\mathrm{C}-2^{\prime}\right.$ and $\left.\mathrm{C}-6^{\prime}\right), 132.6\left(\mathrm{C}-4^{\prime}\right), 135.7$ (C-1'), 144.2 (C4), $193.8(\mathrm{C}=\mathrm{O})$; HRMS (ESI): $m / z[\mathrm{M}+\mathrm{H}]^{+}$calcd for $\mathrm{C}_{10} \mathrm{H}_{10} \mathrm{ClN}_{4} \mathrm{O}: 237.6651$; found: 237.0536 .

1-[5-Amino-1-(4-methoxyphenyl)-1H-1,2,3-triazole-4yl]ethanone (8c): Yield: 46\%; brown solid; $\mathrm{mp} 206.4$ $207.8^{\circ} \mathrm{C}$; IR (KBr): $3324(\mathrm{~N}-\mathrm{H}), 1656(\mathrm{C}=\mathrm{O}) \mathrm{cm}^{-1} ;{ }^{1} \mathrm{H}$ $\mathrm{NMR}\left(\mathrm{CDCl}_{3}, 300 \mathrm{MHz}\right): \delta=2.66\left(\mathrm{~s}, 3 \mathrm{H}, \mathrm{CH}_{3}\right), 3.88(\mathrm{~s}$, $\left.3 \mathrm{H}, \mathrm{OCH}_{3}\right), 5.54\left(\right.$ br s, $\left.2 \mathrm{H}, \mathrm{NH}_{2}\right), 7.07(\mathrm{~d}, J=9.0 \mathrm{~Hz}, 2 \mathrm{H}$, $\mathrm{H}-3^{\prime}$ and $\left.\mathrm{H}-5^{\prime}\right), 7.42\left(\mathrm{~d}, J=9.0 \mathrm{~Hz}, 2 \mathrm{H}, \mathrm{H}-2^{\prime}\right.$ and $\left.\mathrm{H}-6^{\prime}\right) ;{ }^{13} \mathrm{C}$ $\mathrm{NMR}\left(\mathrm{CDCl}_{3}, 75 \mathrm{MHz}\right): \delta=26.2\left(\mathrm{CH}_{3}\right), 55.6\left(\mathrm{OCH}_{3}\right), 115.3$ (C-3' and C-5'), 125.8 (C-3' and C-5'), 129.6, 144.5, 160.5 $(\mathrm{C}=\mathrm{O})$; HRMS (ESI): $m / z[\mathrm{M}+\mathrm{H}]^{+}$calcd for $\mathrm{C}_{10} \mathrm{H}_{13} \mathrm{~N}_{4} \mathrm{O}_{2}$ : 233.2460; found: 233.1031 